\title{
Effects of contextual composition of category triads on semantic interrelatedness among members
}

\author{
LORRAINE A. LOW \\ Framingham State College, Framingham, Massachusetts
}

\begin{abstract}
The influence of the composition of category members during study on the extent of semantic interrelatedness among the members during subsequent recognition was examined. Triads during study contained members at two adjacent levels of taxonomic classification. Members from the superordinate level (e.g., furniture) are considered to be less semantically related than members from the subordinate level (e.g., baby furniture). A target (e.g., crib) was a member that could be contextualized at either level of categorization. The foil during a subsequent recognition test consisted of an encoding triad with a substitute for the target. The substitute was drawn from either the superordinate level or the subordinate level or was unrelated to the target. The relative false-alarm rates for the various substitutes measured the extent of semantic relatedness between the target and other class members. The results confirmed the hypothesis that the semantic interrelatedness of members of the triads during encoding influenced the relatedness of the target to other category members during the recognition phase.
\end{abstract}

In a previous experiment (Low, 1988; Low \& Roder, 1983), the influence of context on category definition of its members was examined. It was expected that category assignment of any particular category member would vary predictably with the remaining members with which it was grouped. The predictions followed from Tversky's (1977) view that the meaning of a category member depends upon the presented set. In that experiment, triads were formed during study. Triad members belonged either to a superordinate level category (e.g., furniture) or to a subordinate level category that was formed by combining the superordinate level category with a simple attribute (e.g., baby furniture). A specific triad containing members from the superordinate level furniture would be crib, stool, and desk. A triad containing members from the subordinate level would be crib, playpen, and highchair. Since crib could be contained in either of the above triads, it was designated the target. The composition of the triads was varied by assigning members at each of these two levels of classification. It was expected that changes in the composition of the triads would be accompanied by changes in the characteristics associated with the particular target.

Current semantic-features theories of taxonomic classification (Medin \& Smith, 1984; Rosch, Mervis, Gray, Johnson, \& Boyes-Braem, 1976) provided the basis for predictions of contextual effects in the previous study (Low, 1988) as well as in the present one. According to these theories, members of a subordinate level are more semantically interrelated than members of the superordinate level. It was therefore predicted by Low (1988)

Address correspondence to Lorraine A. Low, Department of Psychology, Framingham State College, Framingham, MA 01701. that the extent of perceived semantic relatedness between the target and other category members would be determined by contextual variation in the level of triad composition.

In Low's (1988) study, three major study conditions were defined by the composition of the triads: subordinate level (three members from the subordinate category), superordinate level (three members from the superordinate category), and unrelated total (all members from categories unrelated to each other). Three additional encoding triad types were defined by varying combinations of members from the above three basic conditions. Two different mixed triad types, for example, contained two members from the subordinate level and one from the superordinate level. An unrelated-partial triad contained two members from the subordinate level and one member unrelated to the other two.

To measure the extent to which the context defined by the triad influenced the relatedness of the target to other members, a novel procedure-a recognition test-was used. The foil consisted of triad containing a substitute for the target with the other two members remaining the same as in the study phase. The recognition substitute varied in its relationship to the target itself. The substitute for the target during recognition was either a subordinate-level member (e.g., bassinet substituted for crib) or a superordinate-level one (e.g., couch substituted for crib). The number of false alarms during the recognition task measured the similarity of a substitute to the target.

Low's (1988) findings strongly supported the hypothesis that the extent to which the target was semantically related to other category members during recognition varied with the contextual level during encoding. First, with subordinate-level encoding, a subordinate-level substitute 
for the target resulted in a greater number of false alarms than a superordinate-level substitute. For example, if the encoding triad was crib, playpen, and highchair, substituting bassinet for crib yielded many more false alarms than substituting couch. Second, with superordinate-level encoding, there were no differences in the level of the substitute. For example, if the triad was crib, stool, and desk, substituting either bassinet or couch for crib yielded the same number of false alarms. Third, a most interesting finding was that substituting a superordinate-level member during the recognition phase yielded many fewer false alarms with a subordinate-level triad than with a superordinate-level one. Thus, substituting couch for crib yielded fewer false alarms when the triad was crib, playpen, and highchair than when the original triad was crib, desk, and stool.

The present study was an attempt to replicate the findings of Low (1988). The four encoding conditions of this study were the same as in the previous study. They included the following triad types: superordinate-level, subordinate-level, unrelated-total, and unrelated-partial. The two mixed-level conditions were eliminated from this experiment. A second change in this experiment was the inclusion of an unrelated substitute. This substitute served as a baseline for comparison of the superordinate- and subordinate-level substitutes.

\section{METHOD}

\section{Subjects}

Forty Framingham State College student volunteers participated in the experiment.

\section{Design and Materials}

Forty-eight sets of category triads were used. A set consisted of triads for each encoding condition and the given substitute for a particular category. A representative set is presented in Table 1 . The assignment of a particular set to an experimental condition was randomly determined, with a particular set used only once. The four encoding conditions were superordinate-level, subordinate-level, unrelated-partial, and unrelated-total. The three recognition substitutes were drawn from the superordinate level or the subordinate level or were unrelated to the target. On one-fourth of the trials, the same triad was presented again during recognition. A 4 (encoding) $\times 4$ (recognition substitute) within-subjects design was implemented. The 16 different treatment combinations, with three triads for each combination, resulted in $\mathbf{4 8}$ trials for study and for test.

\section{Procedure}

The procedure was similar to that used by Low (1988). The random assignment of treatments to trials in study and test was restricted so that each condition was represented within each block of 16 trials. Each triad was typed on an index card and presented separately for $5 \mathrm{sec}$. In the study period, the triad members were positioned randomly one under the other. Subjects were tested individually and instructed to try to remember the words presented. Recognition testing followed immediately. Position of triad members on the index card was rerandomized for test. The subjects were instructed to look at the card and respond "yes" if every member of the presented triad had been seen previously and "no" if one of the three was new.

\section{RESULTS}

The mean number of false alarms and hits for each treatment combination is presented in Table 2. An analysis of variance for the number of false alarms was performed on the 4 (encoding) $\times 3$ (recognition substitute) withinsubjects design. Encoding was not statistically reliable. Recognition substitute was statistically reliable $[F(2,78)$ $=19.04, p<.01, M S e=0.34]$. The Newman-Keuls test was used for all specific comparisons in the experiment, with significant level set at .05 . The mean numbers of false alarms for recognition substitutes were 0.73 , 0.50 , and 0.33 for the subordinate-level, superordinatelevel, and unrelated substitutes, respectively. All three comparisons yielded significant differences. The number of false alarms diminished with the decrease in similarity of the substitute to the target. The greater false-alarm rate for the subordinate-level substitute than for the superordinate-level one replicates the findings of Low (1988) and indicates a higher degree of semantic similarity to the target for the former than for the latter. The finding of significantly fewer false alarms with the unrelated substitute than with the superordinate-level substitute suggests that the superordinate-level substitute was encoded as semantically related to the target.

The encoding $\times$ recognition substitute interaction was statistically significant $[F(6,234)=5.89, p<.01, M S e$ $=0.37]$. Specific comparisons yielded a reliably greater number of false alarms for the subordinate than for the superordinate substitute with subordinale-level encoding. The difference replicates these findings in Low's (1988) study. As in the previous study, a similar difference was found for the unrelated-partial encoding condition. The latter finding lends support to the view that two members from the subordinate level are sufficient to encode at that level. With superordinate-level encoding, the false-alarm rate was higher for the superordinate-level substitute than for the subordinate-level one. The differences were not

Table 1

Representative Stimulus Set

\begin{tabular}{|c|c|c|c|c|c|c|}
\hline \multicolumn{5}{|c|}{ Encoding Triad } & \multirow{2}{*}{\multicolumn{2}{|c|}{ Recognition Substitute }} \\
\hline \multirow{2}{*}{$\begin{array}{c}\text { Subordinate } \\
\text { (Motor Vehicles) }\end{array}$} & \multirow[b]{2}{*}{ Mixed } & \multirow{2}{*}{$\begin{array}{l}\text { Superordinate } \\
\text { (Vehicles) }\end{array}$} & \multirow[b]{2}{*}{ Unrelated-Partial } & \multirow[b]{2}{*}{ Unrelated-Total } & & \\
\hline & & & & & Subordinate & Superordinate \\
\hline Taxi & Taxi & Taxi & Taxi & Taxi & Truck & Bicycle \\
\hline Bus & Bus & Airplane & Bus & Doll & & \\
\hline Limousine & Subway & Subway & Doll & Coal & & \\
\hline
\end{tabular}

Note-In this example, the target was taxi. Unrelated-Partial = two members from the subordinate category and one member from an unrelated category. Unrelated-Total $=$ three members from unrelated categories. 
Table 2

Mean Number of False Alarms for Each Encoding Condition, Recognition Substitute, and Same Condition (Hits)

\begin{tabular}{lcccc}
\hline \multirow{2}{*}{$\begin{array}{c}\text { Recognition } \\
\text { Substitute }\end{array}$} & \multicolumn{4}{c}{ Encoding Condition } \\
\cline { 2 - 5 } \multicolumn{1}{c}{ Subordinate } & Superordinate & Unrelated-Partial & Unrelated-Total \\
Unrelated & 0.15 & 0.32 & 0.55 & 0.30 \\
Superordinate & 0.35 & 0.85 & 0.40 & 0.40 \\
Subordinate & 0.98 & 0.52 & 0.82 & 0.60 \\
Same (Hits) & 2.55 & 2.48 & 2.25 & 1.95 \\
\hline
\end{tabular}

Note-Unrelated-Partial $=$ two members from the subordinate category and one member from an unrelated category. Unrelated-Total = three members from unrelated categories.

statistically reliable, however. In both the present experiment and the previous one, therefore, the subordinatelevel substitute yielded a greater number of false alarms than the substitute from the superordinate level when the encoding triad was from the subordinate or unrelatedpartial encoding conditions, but not when the encoding triad was from the superordinate level. These findings are consistent with the view that the composition of the triad influenced the encoding of the target. When the encoding was at the subordinate level, only the subordinate-level substitutes belonged to the category. At the superordinate level, both substitutes were members of the category.

Specific comparisons of the superordinate-level and unrelated substitutes for the various encoding conditions yielded only one significant difference. With superordinate-level encoding, a higher rate of false alarms was produced with the superordinate-level substitute than with the unrelated substitute. This difference, however, could be accounted for by the greater number of false alarms with the superordinate-level substitute than with the subordinate-level one for the superordinate-level encoding condition (see Table 2).

A higher false-alarm rate for the subordinate-level than for the superordinate-level encoding condition with a subordinate-level substitute was reliable. The direction of the difference for the unrelated-partial triad type was also in the expected direction but was not statistically significant. These results lend support to the view that members from the subordinate level were encoded as more similar to each other than members from the superordinate level.

Reliably fewer false alarms were made when the subordinate-level or unrelated-partial condition was compared with the superordinate one, given a substitute from the superordinate level. This finding is consistent with that of Low (1988) and strengthens the view that when the triad was composed of subordinate-level members, superordinate recognition substitutes were judged as highly dissimilar to the target. The low false-alarm rate for the unrelated-total condition might counter the argument that encoding at the subordinate level was necessary for exclusion of a superordinate-level member. However, the unrelated-total condition produced significantly fewer false alarms than the subordinate-level encoding condi- tion with the subordinate-level member as well (see Table 2). It is therefore reasonable to conclude that the low rate of false alarms for the unrelated-total condition reflects the high criterion level for responding affirmatively when members of the triad are not related.

The $d^{\prime}$ measure was computed for each subject in each encoding condition, using the false-alarm rate for subordinate-level foils. The four-level one-way analysis of variance yielded statistically reliable findings $[F(3,117)$ $=2.70, p<.05, M S e=2.31]$. The only significant specific comparison was between the superordinate-level and unrelated-total encoding conditions. The means of 2.80 and 1.87 , respectively, indicated poorer discrimination for the unrelated-total than for the superordinatelevel encoding condition.

The number of false alarms for the subordinate-level substitute was compared directly with the hit rate (recognition change). The means for each condition are also given in Table 2 . An analysis of variance was performed on the 4 (encoding) $\times 2$ (recognition change) withinsubjects design. Recognition change was highly significant $[F(1,39)=271.19, p<.01, M S e=0.73]$. In this experiment, as in the earlier one (Low, 1988), subjects were discriminating very well between the original triad and the foil. Encoding differences were also statistically significant $[F(3,117)=6.49, p<.01, \mathrm{MSe}=0.49]$. Specific comparisons indicated that the unrelated-total encoding condition yielded fewer hits and false alarms than the subordinate-level encoding condition. This finding strongly suggests that when a category response was not elicited, the criterion for responding in the affirmative was raised.

The encoding $x$ recognition change interaction was statistically significant $[F(3,117)=3.22, p<.05, \mathrm{MSe}$ $=0.44]$. A specific comparison between hits and false alarms for each encoding condition yielded reliable differences, thus providing further evidence that in all conditions subjects were discriminating between the targets and a highly related foil. The only reliably specific differences found for the hit rates were those between unrelated-total encoding and each of the remaining encoding conditions. These findings, along with the $d^{\prime}$ differences, suggest that when there is no possibility of eliciting a categorical response among triads, the individual items are not encoded as well. 


\section{DISCUSSION}

The results indicate that the level of classification associated with a particular triad defined for the target rather precisely the categorical level at which it was encoded. The degree of similarity between the target and other category members during recognition was predictable from the level of encoding of the triad. These findings are consistent with Tversky's (1977) view that categorization is a dynamic process. The basis for judging similarities and differences between members within a category relies heavily on the set. The findings also support the encoding-specificity hypothesis of Tulving (1979). Tulving's view is that the retrieval of information is effective to the degree that the information is compatible with the encoding trace. In the present study the categorical relatedness at the time of recognition was predictable from the information presented at the time of study.

In the present study, the semantic-feature model provided a basis for prediction of encoding differences associated with levels. According to this model, the more subordinate the level of classification, the greater the number of specific overlapping features, and hence the greater the perceived semantic interrelatedness among members. Some of the present findings associated with different levels of classification were compatible with the model. First, subordinate-level encoding followed by a subordinate-level substitute produced a greater number of false alarms than did subordinate-level encoding followed by a superordinate-level substitute. Second, this difference associated with the level of the recognition substitute was absent when encoding was at the superordinate level. These findings replicated those of Low (1988). Third, this difference in semantic interrelatedness as a function of levels resulted in fewer false alarms for the subordinate-level substitute when encoding was at the superordinate level than when it was at the subordinate level. This finding was in the expected direction in the previous study and was statistically significant in the present one.

Other findings of judged groupings did not follow directly from a strict semantic-interrelatedness model. For example, in both experiments fewer false alarms were found with a recognition substitute at the superordinate level when the triad was composed of members from the subordinate level than when the triad was composed of members from the superordinate level. A nested-classification model (Medin \& Smith, 1984) proposes that all defining features at the superordinate level enter into the category definition at the subordinate level of classification. If all features were weighted in deciding category membership, then a superordinate-level substitute should have yielded the same number of false alarms whether the triad was superordinate or subordinate. Some weighted-features models, such as that proposed by Tversky (1977), can more successfully account for this finding. Tversky argued that only those features that are diagnostic in differentiating members from nonmembers are used in classification. Therefore, in a taxonomic classification scheme, those features that differentiate members from nonmembers at the most subordinate level serve as a basis for determining category membership. In the present study, only those features associated with the definition of the conjunctive category were included. When a triad such as crib, highchair, and playpen was presented, only those characteristics associated with baby furniture were considered. Therefore, the member couch was highly discriminable from the remainder of the set.
Other evidence supports this view that only features that are diagnostic of differences between set and nonset members are used in defining class membership. It has been proposed (Cruse, 1977) that when category members are expressed, only contrasting sets within the same level of classification are implied. This view has experimental support. Tversky and Hemenway (1984) found, for example, that when subjects were asked to list characteristics of subordinates such as pine tree, properties such as stems and roots, which are characteristics of trees in general, were not included.

Overall, the present study indicates that subjects are able to effectively use the complex categories formed by combining an attribute with a simple category. This level of categorization is not considered in the system proposed by Rosch et al. (1976), but has been the focus of attention of other investigators (Hampton, 1987).

In conclusion, the present study demonstrated that the context at the time of encoding is a strong predictor of the categorization process. Moreover, the novel procedure introduced (the similarity of the substitute to the target) proved to be an extremely effective measure of judged similarities in the absence of specific instructions to make these judgments. Most of the present findings can be accounted for by a strict semantic-feature-overlap model. Other findings can be explained by assigning stronger weights to those features that discriminate members from nonmembers at the most subordinate level. The present study sug gests that manipulation of context at the time of encoding is an effective means of studying those characteristics that are used in deciding category membership.

\section{REFERENCES}

Cruse, D. A. (1977). The pragmatics of lexical specificity. Journal of Linguistics, 13, 153-164.

HAMPTON, J. A. (1987). Inheritance of attributes in natural concept conjunctions. Memory \& Cognition, 15, 55-71.

Low, L. A. (1988). Contextual effects on encoding and recognition of category members subsumed by two levels of classification. Bulletin of the Psychonomic Society, 26, 301-304.

Low, L. A., \& Roder, B. J. (1983). Semantic relations between encoding and retrieval in cued recall. Memory \& Cognition, 11, 651-659.

Medin, D. L., S Sitr, E. E. (1984). Concepts and concept formation. Annual Review of Psychology, 35, 113-138.

Rosch, E., Mervis, C. B., Gray, W., Johnson, D., \& BoyesBraem, P. (1976). Basic objects in natural categories. Cognitive Psychology, 8, 382-439.

Tulving, E. (1979). Relation between encoding specificity and levels of processing. In L. S. Cermak \& F. I. M. Craik (Eds.), Levels of processing in human memory (pp. 405-428). New York: Halsted Press.

TVersky, A. (1977). Features of similarity. Psychological Review, 84, 327-352.

TVerSKY, B., \& HemenWAY, K. (1984). Objects, parts, and categories. Journal of Experimental Psychology: General, 113, 169-193.

(Manuscript received for publication December 30, 1987.) 